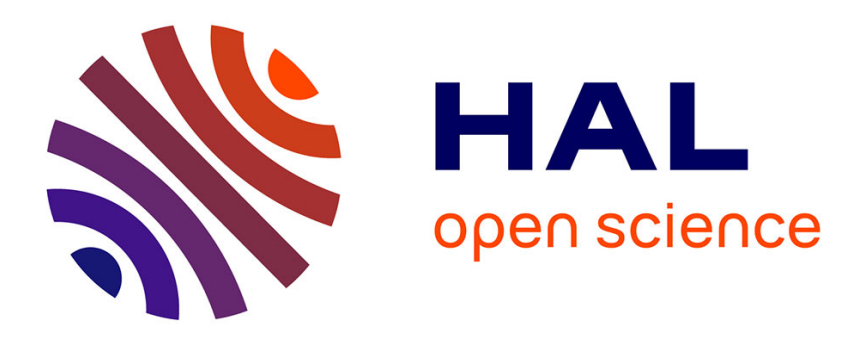

\title{
Robust green Wireless Local Area Networks: A matheuristic approach
}

Rosario Garroppo, Maria Grazia Scutellà, Fabio d'Andreagiovanni

\section{To cite this version:}

Rosario Garroppo, Maria Grazia Scutellà, Fabio d'Andreagiovanni. Robust green Wireless Local Area Networks: A matheuristic approach. Journal of Network and Computer Applications (JNCA), 2020, 163, pp.102657. 10.1016/j.jnca.2020.102657 . hal-02946275

\section{HAL Id: hal-02946275 \\ https://hal.science/hal-02946275}

Submitted on 4 Jan 2021

HAL is a multi-disciplinary open access archive for the deposit and dissemination of scientific research documents, whether they are published or not. The documents may come from teaching and research institutions in France or abroad, or from public or private research centers.
L'archive ouverte pluridisciplinaire $\mathbf{H A L}$, est destinée au dépôt et à la diffusion de documents scientifiques de niveau recherche, publiés ou non, émanant des établissements d'enseignement et de recherche français ou étrangers, des laboratoires publics ou privés. 


\title{
Robust Green Wireless Local Area Networks: a matheuristic approach
}

\author{
Rosario G. Garroppo ${ }^{a, *}$, Maria Grazia Scutellà ${ }^{\text {, Fabio D'Andreagiovannic }}{ }^{c}$ \\ ${ }^{a}$ Dipartimento di Ingegneria dell'Informazione, Università di Pisa, Italy \\ email: rosario.garroppo@unipi.it \\ ${ }^{b}$ Dipartimento di Informatica, Università di Pisa, Italy \\ email:scut@di.unipi.it \\ ${ }^{c}$ French National Center for Scientific Research (CNRS), Heudiasyc UMR 7253, Sorbonne \\ Universités, Université de Technologie de Compiègne, France \\ email: fabio.dandreagiovanni@utc.fr
}

\begin{abstract}
We consider the problem of designing a Wireless Local Area Network according to a Green paradigm (GWLAN), i.e. minimizing the power consumption of the network by powering-on just a subset of access points and associating users with the powered-on access points. To protect the system against fluctuations in the data rate transmission between users and access points, which naturally affect the problem because of uncertainty in measuring the exact user position and because of wireless propagation conditions, we address the robust extension of this problem, i.e. the Robust Green Wireless Local Area Networks Problem (RGWLAN), and we propose some robust matheuristics to solve it. Such matheuristics are based on two mathematical models to RGWLAN, i.e. a model based on a generalization of the classical $\Gamma$-Robustness framework, and a model exploiting the Multiband Robustness paradigm. The models are enriched by means of a preprocessing powering-on heuristic, aimed at fixing some access points as powered-on, and a reallocation algorithm, aimed at reallocating users to access points once the model solution has been computed, in order to enhance its degree of robustness. The aim is to achieve a good compromise between power saving, guaranteed level of robustness and required computational time.

The results of an extensive computational analysis show that the proposed matheuristics solve the RGWLAN in a very efficient way both in terms of power consumption and computational time. Also, they are able to guarantee a high level of robustness with respect to the uncertainty in the user positions. This trend is particularly evident when the users to access points ratio is low, i.e.
\end{abstract}

\footnotetext{
* Corresponding author rosario.garroppo@unipi.it

Dip. di Ingegneria dell'Informazione - Università di Pisa

Via Caruso, 16 - 56122 - Pisa, ITALY

Tel. : +39050 2217621 Fax.: +39050 2217522
} 
when the offered traffic to the wireless local area network is scarce, which is the most suitable and relevant scenario for the power consumption optimization.

Keywords: Green Wireless Local Area Network, User Position Uncertainty, Robust Optimization, Multiband Robust Optimization, Matheuristic.

\section{Introduction}

In recent times, telecommunications networks have become an essential infrastructure all around the world, providing services running on devices such as personal computers and smartphones that now represent a fundamental part of our everyday life. In particular, Internet connectivity constitutes one of the most requested services and a very widespread way to provide it is represented by Wireless Local Area Networks (WLANs). A WLAN is a network that employs wireless links in order to connect multiple devices located in a limited area (e.g., an office, an apartment, a park) between them and to Internet. Specifically, it is composed of a set of Access Points (APs) that provide wireless connectivity to a set of User Terminals (UTs), often based on Wi-Fi standards. Compared to wired networks, WLANs offer advantages in terms of cost savings, due to easier installation procedures, and in terms of access and flexibility, since the users can freely move around the area covered with the service without losing the connection. However, WLANs present also a major disadvantage, namely the variable performance of wireless connections: as one can commonly experience, the data rate associated with a connection typically varies over time, also depending on the position and movement of the users. This translates into uncertainty about the value of data rate that the user is able to obtain. Another problematic is represented by the WLANs power consumption: in order to guarantee high data rates, WLANs typically install a high number of APs which, however, are often powered-on but idle. This may lead to a waste of energy that is nowadays not acceptable both to contain costs and be environmentally friendly. For example, in the context of dense WLANs, the authors in Debele et al. (2015) show that, by dynamically activating only the number of APs that is strictly needed to the current traffic, then up to $59.1 \%$ of power can be saved with respect to the absence of any strategy.

To deal with both the power containment and the data rate uncertainty questions, in this paper we consider the Robust Green Wireless Local Area Networks Problem (RGWLAN). This problem, introduced in the studied form in Garroppo et al. (2016b), consists in minimizing the power consumption of a WLAN by powering-on just a subset of the installed APs and associating the UTs to powered-on APs, and simultaneously taking into account the uncertainty of the data rates between UTs and APs. In the context of GWLAN, in fact, it is of paramount importance to protect the system against natural fluctuations in the network performance that occur over short periods of time and lead to tricky reductions in data rates. We stress that designing green networks is a major question not only for WLANs, but also for telecommunications 
networks in general, and a lot of work has been done on the subject. We refer the reader to Bianzino et al. (2012), Li et al. (2011) and Wang et al. (2012) for exhaustive surveys about opportunities and challenges arising in green networking and wireless communications. On the other hand, less work has been done in optimizing the WLAN design while taking into account uncertainty issues. Specifically, two Robust Optimization (RO) models have been recently proposed to formulate the RGWLAN problem, i.e. to achieve both power saving and robustness against the data rate uncertainty in the context of WLANs. The first robust model has been proposed in Garroppo et al. (2016b) by generalizing the classical $\Gamma$-Robustness of Bertsimas and Sim (Bertsimas et al. (2011).Bertsimas and Sim (2004)). There, the phenomenon of the data rate fluctuation has been addressed by considering the uncertainty in measuring the exact user position in the area, also due to the user mobility, and the impact of the wireless propagation conditions on the data rates. The second robust model, on the other hand, exploiting the Multiband Robust Optimization (MRO) technique (Büsing and D'Andreagiovanni (2012), Bauschert et al. (2014)), has been preliminary investigated in D'Andreagiovanni et al. (2018). With respect to Garroppo et al. (2016b), MRO is used to model the user position uncertainty, while adopting the $\Gamma$-Robustness to model the uncertainty due to the wireless propagation conditions. With respect to the canonical MRO model proposed in Büsing and D'Andreagiovanni (2012), the presence of two distinct uncertain events that are mutually dependent has been considered, thus extending the theory of MRO.

\subsection{Paper contribution}

To the best of our knowledge, our short conference papers Garroppo et al. (2016b) and D'Andreagiovanni et al. (2018) are the only works in the literature addressing the RGWLAN. As previously mentioned, they propose mathematical formulations to the problem, to guarantee an optimal power consumption of the system while taking into account the uncertainty of the UT-AP data rates. Due to the computational time complexity of RGWLAN, which is NP-Hard, such mathematical formulations may require a large amount of time to be solved exactly. Therefore, in this paper we extend and complete the results in Garroppo et al. (2016b) and D'Andreagiovanni et al. (2018) by consolidating the modelling aspects, and presenting efficient robust mathematical optimization-based heuristics, or matheuristics, to RGWLAN, with the aim of achieving a good compromise among power saving, guaranteed level of robustness and required computational time.

Specifically, the proposed matheuristics are designed by enriching the two robust models in D'Andreagiovanni et al. (2018) and Garroppo et al. (2016b) by means of two heuristic algorithms:

1. a preprocessing powering-on heuristic, aimed at discovering APs to turnon, and fixing them as powered-on within the model statement; the goal is to accelerate the model solution, so reducing the computational effort, without increasing too much the optimal power consumption guaranteed by the mathematical models alone; 
2. a reallocation algorithm, aimed at reallocating UTs to APs once the model solution has been computed; the goal is to enhance, at a negligible computational cost, the degree of robustness against the data rate uncertainty experienced by the users within the area, without deteriorating the system power consumption too much.

The main contributions of this paper can be summarized as follows.

- We extend the theory of the canonical MRO models proposed in Büsing and D'Andreagiovanni (2012) by considering the presence of two distinct uncertain events that are mutually dependent.

- We enhance the research on the RGWLAN by enriching the two robust models in D'Andreagiovanni et al. (2018) and Garroppo et al. (2016b) with some heuristic devices, so converting the robust mathematical models into robust matheuristics.

- We investigate the efficiency and the efficacy of the proposed matheuristics by means of a wide computational experience. We show that, when the UTs to AP ratio is low, then the matheuristics based on Multiband Robust Optimization are very efficient in terms of power saving and computational time, and guarantee a high level of robustness in most cases. When the UTs to AP ratio increases, instead, the matheuristics are less efficient: the ones based on the more classical $\Gamma$-Robustness show a good level of robustness, but they may have more difficulties in solving the problem.

Regarding the paper organization, Section 2 provides an overview on related works. The GWLAN problem, i.e. when uncertainty issues are not taken into account, is presented in Section 3 together with a mathematical formulation, while its robust extension, that is RGWLAN, is described in Section 4 together with the mathematical models mentioned before. Section 5 presents the powering-on heuristic and the reallocation algorithm. Section 6 discusses some architectural aspects for implementing GWLANs and gives ideas on how the proposed algorithms can be integrated in actual Wi-Fi networks. Section 7 reports the results of extensive computational experiments, testing the matheuristics in different network scenarios, while Section 8 concludes the paper.

\section{Related Work}

As outlined before, the problem addressed in this paper, i.e. RGWLAN, has been never studied in the literature with the exceptions of the preliminary works Garroppo et al. (2016b) and D'Andreagiovanni et al. (2018). Therefore, here we shall review some related papers, mainly addressing optimization issues in WLANs.

Since the UT-AP association in WLAN systems is a critical design decision in our study, the focus will be on papers dealing with such a relevant decision 
issue. The optimal assignment of UTs to APs is in fact very relevant in the context of WLANs, and it has received a consistent attention in the literature. One of the first works presenting an optimization model for determining an optimal association in WLANs is Bejerano et al. (2007), which also takes into account a max-min fair bandwidth allocation among the users and proposes approximation algorithms for the online solution of the problem. In Li et al. (2008), approximation algorithms are instead proposed for periodical offline optimization in order to improve the fairness of bandwidth allocation. In Amer et al. (2018), the UT-AP association is operated through a centralized optimization approach based on an assignment problem including a logarithmic objective function to represent data rate fairness. The problem is solved through a simple multi-start local search heuristic based on identifying new solutions by allowing only one single UT to change its serving AP. In Li et al. (2013), the problem of a fair association in WLANs is modelled as a hard nonlinear integer programming problem which is addressed through a four-stage heuristic which first relaxes the integer variables representing the UT-AP association, then solves the corresponding easier continuous problem, relies on a rounding strategy to derive an integral association, and finally operates a load redistribution to define a feasible (non-optimal) design solution. In Tang et al. (2016), constraints expressing the minimum data rate requested by the UTs are included in the optimization model, discussing how they make the solution process more difficult and proposing two heuristic algorithms for the solution of the related optimization problem. What is common to all the previously cited works is that they consider the problem of modelling and designing a WLAN, or some related wireless networks, 1) without assuming a green networking perspective, i.e. trying to limit the power consumption of the network, and 2) without taking into account the natural uncertainty that affects the data rates, namely the fact that the exact value of the data rates is not known when the problem is solved, due to uncertain channel conditions and user mobility.

The question of designing WLANs under a green paradigm, i.e. GWLANs, has been considered in several works. In Garroppo et al. (2016a), the authors computationally evaluate how different power consumption models impact on the solutions produced solving the green WLAN network design problem. In Zhang et al. (2018), a tabu-search algorithm is proposed to solve the problem of minimizing the power consumption by turning-off subsets of APs and assigning their users to other active APs without compromising the Quality-of-Service. In $\mathrm{Wu}$ et al. (2018), a two-stage heuristic algorithm is proposed for the power consumption minimization: firstly, a limited number of AP-UT assignments is eliminated to reduce the traffic congestion and turn-off as many idle APs as possible; then, the unassigned UTs are reassigned to some APs by trying to improve the energy efficiency. In Gendron et al. (2016) an efficient branch-andcut algorithm based on the principles of Benders' Decomposition is proposed to tackle large scale instances. However, no paper dealing with a green WLAN optimal design jointly addresses the uncertainty of the data rates, except our short conference papers Garroppo et al. (2016b) and D'Andreagiovanni et al. (2018), where robust exact approaches have been outlined and preliminary investigated. 
For a recent survey on Robust Optimization techniques we refer the interested reader to Gabrel et al. (2014). See also Koster and Poss (2018) for an overview on Robust Combinatorial Optimization. We remark that Robust Optimization techniques have been applied to address uncertainties arising in telecommunications networks other than WLANs. At this regard we mention Büsing et al. (2017), in the context of optical networks, Di Puglia Pugliese et al. (2019), for solving the resource constrained shortest path problem with uncertain data, and Claßen et al. (2013), for more general wireless network planning. We also remark that, though matheuristics are nowadays a well-consolidated solution approach for hard-to-solve optimization problems (see e.g. the surveys Archetti and Speranza (2014) and Talbi (2016)), to the best of our knowledge they have not been employed so far in optimizing the GWLANs.

\section{GWLAN: problem and mathematical model}

A GWLAN system is composed of a set $\mathcal{J}$ of deployed APs that can serve a set $\mathcal{I}$ of UTs. The traffic demand $w_{i}$ of each UT $i$ must be satisfied by exactly one AP. The power $P_{j}$ consumed by the generic $\operatorname{AP} j$ can be essentially ascribed to two major components (Garroppo et al. (2016a)): 1) a fixed component $p_{j}^{o}$, which is bound to the mere fact that the device is powered-on; 2) a variable component $a_{j}$, which accounts for the so-called "airtime", i.e. the fraction of time the device is either transmitting or receiving frames. The component $a_{j}$ is weighted by a constant "wireless" factor $p^{w}$, which accounts for the power drain of the radio frontend for the transmission and reception operations (see Garroppo et al. (2016b) for more details), and the overall power consumption $\overline{P_{j}}$ is then: $P_{j}=p_{j}^{o}+p^{w} a_{j}, \quad \forall j \in \mathcal{J}$.

The other parameters characterizing the GWLAN system are the $r_{i j}$, i.e. the data rate available between the $\mathrm{UT} i$ and the $\operatorname{AP} j$, for $i \in \mathcal{I}$ and $j \in \mathcal{J}$. They depend on the physical properties of the system, such as the position of the UT $i$ with respect to the AP $j$. To keep the notation simpler, we shall assume that the links are symmetric, i.e. $r_{i j}=r_{j i}, \forall i \in \mathcal{I}, j \in \mathcal{J}$.

The GWLAN problem consists in deciding which APs to power-on and to which powered-on AP to assign each UT, so as to satisfy the demand of each UT and the capacity constraint of each AP. The goal is to minimize the overall power consumption of the system. Notice that, in this context, the data rates $r_{i j}$ are assumed to be exactly known. In other words, their uncertainty is not taken into account.

By introducing the following two sets of binary variables:

- $x_{i j}$, set to 1 if $\mathrm{UT} i$ is assigned to $\operatorname{AP} j$ and to 0 otherwise, $\forall i \in \mathcal{I}, j \in \mathcal{J}$,

- $y_{j}$, set to 1 if $\mathrm{AP} j$ is powered-on and to 0 otherwise, $\forall j \in \mathcal{J}$,

the considered optimization problem can be formulated in terms of the following Binary Linear Programming (BLP) model, initially proposed in Garroppo et al. 
(2016b):

$$
\begin{aligned}
& z=\min \sum_{j \in \mathcal{J}} P_{j}=\min \sum_{j \in \mathcal{J}}\left[p_{j}^{o} y_{j}+p^{w} \sum_{i \in \mathcal{I}} \frac{w_{i}}{r_{i j}} x_{i j}\right], \\
& \sum_{j \in \mathcal{J}} x_{i j}=1 \quad i \in \mathcal{I} \\
& \sum_{i \in \mathcal{I}} \frac{w_{i}}{r_{i j}} x_{i j} \leq y_{j} \quad j \in \mathcal{J} \\
& x_{i j} \in\{0,1\} \quad i \in \mathcal{I}, j \in \mathcal{J} \\
& y_{j} \in\{0,1\} \quad j \in \mathcal{J} \text {, }
\end{aligned}
$$

where the airtime $a_{j}$ is expressed in terms of the variables $x_{i j}: a_{j}=\sum_{i \in \mathcal{I}} \frac{w_{i}}{r_{i j}} x_{i j}$. For sake of clarity, we observe that for $i \in \mathcal{I}$ and $j \in \mathcal{J}$, if $r_{i j}=0$ then $x_{i j}=0$. This condition physically means that we cannot associate a user $i$ with an AP $j$ if the link $(i, j)$ has a null data rate.

In this model, constraints (2) express that each UT must be assigned to exactly one AP, whereas constraints (3) express the capacity of the APs and also ensure that no UT is assigned to powered-off APs. The model is characterized by $|\mathcal{J}|+|\mathcal{I}||\mathcal{J}|$ binary variables and by $|\mathcal{I}|+|\mathcal{J}|$ constraints, plus the ones defining the variable domain. For a general overview on assignment based problems and models, we refer to Burkard et al. (2009).

\section{RGWLAN: problem and mathematical models}

In GWLAN, the assumption is made that the data rates $r_{i j}$ are exactly known when the problem is solved. However, in practice this is not true, since $r_{i j}$ depend on the exact position of the users in the area, whose estimate is naturally subject to inaccuracies, also due to their movements during the estimation phase. Furthermore, $r_{i j}$ depend also on the propagation conditions of the wireless channel, which are hard to exactly know a priori. The data rates $r_{i j}$ are thus naturally subject to uncertainty.

In order to take into account the uncertainty in the user position, we assume that the users can be positioned in diverse annuli areas. Specifically, each user can be situated in an annulus whose ray is not exactly known but belongs to the range $\left[0, \rho^{\max }\right]$, and could be in any position within it. Following the MRO paradigm (Büsing and D'Andreagiovanni (2012), Bauschert et al. (2014)), the Multiband Robust Optimization model presented in D'Andreagiovanni et al. (2018) partitions the overall range $\left[0, \rho^{\max }\right]$ into subranges that correspond to a set $B$ of so-called bands. Each band models a distinct range of deviation of the uncertain data and, in the case of RGWLAN, represents a different class of uncertainty of the user position. The diversity can be due, for example, to a different speed/mobility of the user or to particular difficulties in the estimation of the user location. Specifically, in our computational study we considered 4 classes of user position uncertainty corresponding to 4 deviation bands: 
1. $b=0$ - certain position, i.e. $\rho=0$,

2. $b=1$ - low uncertainty: the user position is in the annulus area defined by $\rho=\left(0, \rho_{1}\right]$,

3. $b=2$ - medium uncertainty: the user position is in the annulus area defined by $\rho=\left(\rho_{1}, \rho_{2}\right]$,

4. $b=3$ - high uncertainty: the user position is in the annulus area defined by $\rho=\left(\rho_{2}, \rho_{3}\right]$,

where $0=\rho_{0}<\rho_{1}<\rho_{2}<\rho_{3}=\rho^{\max }$.

We assume that the position of at most $H_{b} \geq 0$ UTs may belong simultaneously to the class of uncertainty $b$ with $b \geq 1$. Additionally, we distinguish users whose position is certain (class $b=0$ ) and whose wireless channel conditions vary and lead to a variation in the data rate: we assume that at most $H_{0}$ UTs may belong to this category. Finally, we assume that at most $K$ UTs are subject to any kind of uncertainty (due to uncertain position or to wireless propagation uncertainty, discussed below).

Concerning the wireless propagation uncertainty, the actual values $r_{i j}$ are also influenced by variations in the wireless propagation conditions, due to fading phenomena that are really hard to precisely assess. For these phenomena, the Rayleigh model is widely used in the literature (Tse and Viswanath (2005)) and serves as the worst case for a broad class of fading distributions. We consider the case where the $f$-quantile of the Rayleigh fading varies within the interval, or monoband, $\left[f_{L}, \bar{f}\right]$, where $\bar{f}=1-e^{-\frac{\pi}{4}}$ is the nominal quantile, leading to the average value of the fading channel. The parameter $f$ is equal to $\bar{f}$ in case of no fluctuations, while it is set to $f_{L}$ in case of channel fluctuation, since $f_{L}$ represents the worst scenario under the considered uncertainty model.

According to what stated before, for each UT-AP couple $(i, j)$, the data rate depends upon the position of user $i$ and the propagation condition between $i$ and $j$, and thus depends upon the deviation band $b$ and the fluctuation in the Rayleigh fading. We thus denote by $r_{i j}\left(b, f_{L}\right)$ the function representing the data rate for $(i, j)$ when the UT $i$ belongs to the position band $b, b \geq 1$, and the worst Rayleigh fading deviation occurs. Analogously, $r_{i j}\left(0, f_{L}\right)$ denotes the data rate for the case of certain user position, i.e. band $b=0$, and worst fading case. We also introduce the notation $\bar{r}_{i, j}=r_{i, j}(0, \bar{f})$ to denote the nominal value of the data rate for $(i, j)$, i.e. when the user position is certain and no fading fluctuation occurs.

In order to state the robust counterpart of the constraint (3) corresponding to an AP $j \in \mathcal{J}$, let us associate binary variables $q_{i j}^{b}$ with each UT $i$ and band $b$ : $q_{i j}^{b}$ is set to 1 if either the position of the UT $i$ belongs to the band $b \geq 1$, or the position of $i$ is certain $(b=0)$ but the fading channel is subject to fluctuation; it is set to 0 otherwise. The case of UTs with uncertain position whose related data rates are not subject to channel fluctuation is not modelled here, since it is not significant in this context.

By using these additional variables, the robust version of each constraint (3) contains an inner BLP problem, which gives the maximum (i.e., the worst case) value that the left-hand-side may achieve under the considered uncertain 
framework. Precisely, the (nonlinear) robust capacity constraint for each AP $j \in \mathcal{J}$, that includes the maximization of the deviation by means of the variables $q_{i j}^{b}$, writes as:

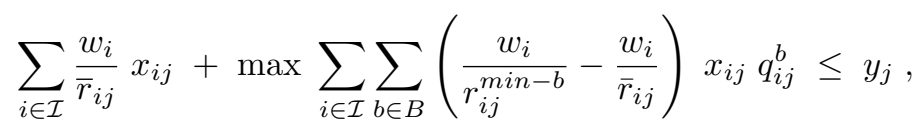

where $r_{i j}^{\text {min-b}}$ denotes the worst value assumed by the data rate function $r_{i j}\left(b, f_{L}\right)$ for UT $i$ and band $b$. Note that when $\sum_{b \in B} q_{i j}^{b}=0$ (i.e. the position of UT $i$ is certain and the related fading channel does not fluctuate), in the left-hand-side we get the fraction $\frac{w_{i}}{\bar{r}_{i, j}}$, using the nominal data rate. Also observe that the values in the denominators in (6) are not decision variables. Furthermore observe that, to satisfy relation $(6), x_{i j}=0$ when $\bar{r}_{i, j}=0$ or/and $r_{i j}^{\text {min }-b}=0$.

For each $j \in \mathcal{J}$, the feasible set of the inner maximisation problem is described by the following set of constraints, where (8) states that at most $K$ users may be subject to uncertainty, assuming $K<\sum_{b \in B} H_{b}$ :

$$
\begin{array}{ll}
\sum_{b \in B} q_{i j}^{b} \leq 1 & i \in \mathcal{I} \\
\sum_{i \in \mathcal{I}} \sum_{b \in B} q_{i j}^{b} \leq K & b \in B \\
\sum_{i \in \mathcal{I}} q_{i j}^{b} \leq H_{b} & \\
q_{i j}^{b} \in\{0,1\} & i \in \mathcal{I}, b \in B
\end{array}
$$

The following property holds true:

Proposition 1. The polytope associated with the linear relaxation of $77-10$ is integral.

Proof: Consider the linear relaxation of $77-(10)$, obtained by replacing the integrality constraints 10 by $0 \leq q_{i j}^{b} \leq 1, i \in \mathcal{I}, b \in B$. Observe that constraints $q_{i j}^{b} \leq 1, i \in \mathcal{I}, b \in B$, can be dropped, since they are dominated by (7). To prove the integrality, state the constraints of the resulting relaxation in matrix form $A q \leq b$, where the matrix $A$ and the right-hand-side $b$ are:

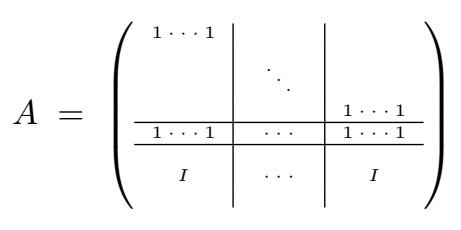

$$
b=\left(\begin{array}{c}
\vdots \\
i \\
\vdots \\
\hline K \\
\hline \vdots \\
H_{b} \\
\vdots
\end{array}\right)
$$


In $A, I$ denotes the identity matrix of size equal to the number of bands, i.e. $|B|$. The matrix $A$ is totally unimodular since: 1$)$ each of its entries is in $\{+1$, $-1,0\}$ and 2) for each subset $M$ of the rows, there exists a partition $\left(M_{1}, M_{2}\right)$ of $M$ such that each column $j$ satisfies: $\left|\sum_{i \in M_{1}} a_{i j}-\sum_{i \in M_{2}} a_{i j}\right| \leq 1$ (see Wolsey (1998), page 50). Since $A$ is totally unimodular and the right-hand-side vector $b$ is integral, it is well-known that the polytope defined by $A q \leq b$ and $q \geq 0$ is integral.

We can then derive a compact Mixed Integer Linear Program (MILP) model to RGWLAN by using the dual problem of the linear relaxation of the maximization deviation problem $(7)-(10)$. Since the linear relaxation of $(7)-(10)$ is feasible and bounded, also its dual is feasible and bounded and, by strong duality, the optimal values of the two problems coincide. We can then replace each inner maximization problem of the original (nonlinear) problem with the corresponding purely linear dual problem, obtaining the following robust (and compact) MILP model:

$$
\begin{array}{ll}
\min \quad & \sum_{j \in \mathcal{J}}\left[p_{j}^{o} y_{j}+p^{w}\left(\sum_{i \in \mathcal{I}} \frac{w_{i}}{\bar{r}_{i j}} x_{i j}+\sum_{i \in \mathcal{I}} \pi_{i}^{j}+K \delta^{j}+\sum_{b \in B} \mu_{b}^{j} H_{b}\right)\right] \\
& \sum_{i \in \mathcal{I}} w_{i} \frac{x_{i j}}{\bar{r}_{i j}}+\sum_{i \in \mathcal{I}} \pi_{i}^{j}+K \delta^{j}+\sum_{b \in B} \mu_{b}^{j} H_{b} \leq y_{j} \quad j \in \mathcal{J} \\
& \pi_{i}^{j}+\delta^{j}+\mu_{b}^{j} \geq\left(\frac{w_{i}}{r_{i j}^{m i n-b}}-\frac{w_{i}}{\bar{r}_{i j}}\right) x_{i j} \quad i \in \mathcal{I}, j \in \mathcal{J}, b \in B(13) \\
& \pi_{i}^{j}, \quad \delta^{j}, \quad \mu_{b}^{j} \geq 0 \\
\text { s.t. }(2),(4), & i(5)
\end{array}
$$

This model includes the robust version (11) and 12 of the objective function and of the capacity constraints, respectively. The additional constraints (13) and the variables (14) are those coming from the classical dualization procedure. Notice that the model has more variables than the ones of the GWLAN model, i.e. the same $|\mathcal{J}|+|\mathcal{I}||\mathcal{J}|$ binary variables plus $3|\mathcal{I}||\mathcal{J}||B|$ continuous variables. Also the number of constraints is greater, being equal to $|\mathcal{J}|+|\mathcal{I}||\mathcal{J}||B|$ plus the constraints defining the variable domain.

The robust framework in Garroppo et al. (2016b) is the special case of the previously described Multiband Robust framework where $b=1$, i.e. the users are positioned in a single annulus. In this case, $H$ is used to denote the maximum number of UTs who may belong to the corresponding uncertainty band. The related mathematical model can be obtained from the Multiband Robust one accordingly (for details we refer to Garroppo et al. (2016b)). Hereafter it will be denoted as the Basic Robust model.

We conclude the section by summarizing in Table 1 the notation used to state the mathematical models. 
Table 1: Notation used in the model formulation

\begin{tabular}{|c|c|}
\hline Symbol & Meaning \\
\hline $\mathcal{J}$ & Set of APs \\
\hline $\mathcal{I}$ & Set of UTs \\
\hline$p_{j}^{o}$ & Fixed power consumption when AP $j$ is powered-on \\
\hline$p^{w}$ & $\begin{array}{l}\text { Constant "wireless" factor for accounting the power consumption } \\
\text { due to transmission and/or reception operations }\end{array}$ \\
\hline$a_{j}$ & $\begin{array}{l}\text { "Airtime" of } \mathrm{AP} j \text {, i.e. fraction of time the } \mathrm{AP} j \text { is either trans- } \\
\text { mitting or receiving frames }\end{array}$ \\
\hline$w_{i}$ & Traffic demand of user $i$ \\
\hline$B$ & Number of bands modelling the uncertainty \\
\hline$b$ & Uncertainty position band, $b=0,1 \ldots, B-1$ \\
\hline$K$ & Maximum number of UTs who may be subject to uncertainty \\
\hline$H_{b}$ & Maximum number of users in the uncertainty band $b$ \\
\hline $\bar{r}_{i, j}$ & $\begin{array}{l}\text { Data rate of link }(i, j) \text { when the user position is certain and no } \\
\text { fading fluctuation occurs }\end{array}$ \\
\hline$r_{i j}^{\min -b}$ & Worst data rate of link $(i, j)$ when UT $i$ belongs to band $b$ \\
\hline$x_{i j}$ & $\begin{array}{l}\text { Binary variable set to } 1 \text { if } \mathrm{UT} i \text { is assigned to } \mathrm{AP} j \text { and to } 0 \\
\text { otherwise }\end{array}$ \\
\hline & Binary variable set to 1 if $\mathrm{AP} j$ is powered-on and to 0 otherwise \\
\hline$q_{i j}^{b}$ & $\begin{array}{l}\text { Additional binary variable set to } 1 \text { if the channel of }(i, j) \text { is subject } \\
\text { to fluctuation when UT } i \text { is in band } b \text {, and set to } 0 \text { otherwise }\end{array}$ \\
\hline$\pi_{i}^{j}, \delta^{j}, \mu_{b}^{j}$ & Dual variables for the linear relaxation of $(7 p-\sqrt{10}$ \\
\hline
\end{tabular}

\section{The powering-on heuristic and the reallocation algorithm}

Based on some preliminary computational results, especially in dense WLAN networks, we have proposed two heuristic tools to improve the performance of the Basic Robust and of the Multiband Robust models presented in Section 4 .

\subsection{The powering-on heuristic}

We have observed that, depending on how UTs and APs are distributed in the area, there may exist a particular set of APs, hereafter denoted as $\mathcal{J}_{O N}$, which can potentially serve a lot of users. Trying to power-off the APs in this set often has the effect to spend time for computing an optimal solution that offers a negligible power consumption gain with respect to the solution where the APs in $\mathcal{J}_{O N}$ are always powered-on. Therefore, it may be convenient to impose the powering-on of the APs in $\mathcal{J}_{O N}$. These powering-on decisions can be fixed within the robust models by setting $y_{j}=1 \forall j \in \mathcal{J}_{O N}$. In this manner, the solver looks for an optimal solution without modifying the setting of these variables. The number of the variables considered during the optimization process is then reduced and, consequently, the computational time $(\mathrm{CT})$ decreases. On the 
opposite, to impose the opening of the APs in $\mathcal{J}_{O N}$ may lead to an increased average power consumption (PC) compared to the optimal one returned by solving the robust models exactly. Indeed, the performance analysis in Section 7 shows that this heuristic tool allows one to achieve a good trade-off between $\mathrm{PC}$ increase and CT shortening.

The powering-on heuristic is detailed in Algorithm 1 . For each AP $j$, the algorithm first evaluates the number of users who can be potentially associated with $j$, denoted as $C_{j}$ (see lines 4-6). A user $i$ can be potentially associated with $j$ if the nominal data rate $\bar{r}_{i j}$ is other than zero. Then, the AP $j$ is powered-on, i.e. $y_{j}$ is fixed to 1 within the robust models, if $C_{j}$ is greater than or equal to a given threshold $T$ (lines 8-10). In our computational experience we set $T=0.80|\mathcal{I}|$, i.e. the AP can potentially serve at least the $80 \%$ of the UTs. This value has been suggested by some preliminary tests, aimed at discovering a good trade-off between the computational time required to solve the optimization model when $T=0$ (according to which all APs are always powered-on, and so the corresponding decisions are fixed within the model) and the power saving corresponding to the setting $T=|\mathcal{I}|$ (according to which no AP is opened a priori, by allowing maximum flexibility to the optimization solver). The powering-on heuristic works in $O(|\mathcal{I}||\mathcal{J}|)$ time, and therefore it is theoretically very efficient.

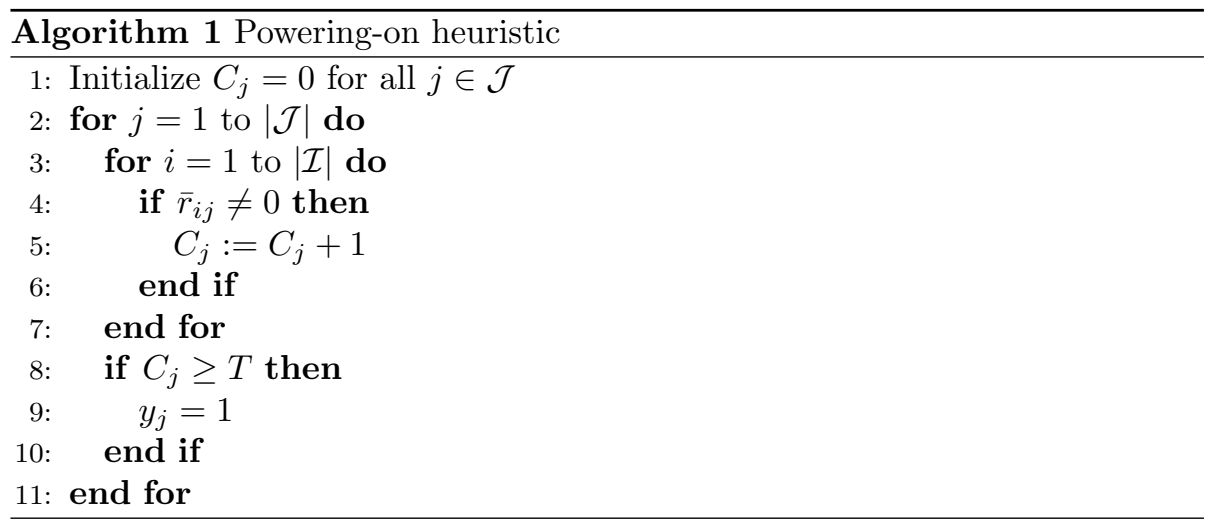

\subsection{The reallocation algorithm}

Preliminary computational results have put in evidence that, in some scenarios, the solutions of the robust models can show a low level of robustness against the user position uncertainty. Specifically, by considering the association UT-AP in such solutions, and simulating the user positions in the different uncertainty bands, often unfeasibility may occur. Precisely, we have observed that there are two main reasons that may lead to unfeasibility:

- the simulated position of a UT $i$ with respect to the assigned AP $j$ is such that the realized data rate between $i$ and $j$ is equal to 0 ; in this case, 
the computed solution is indeed unfeasible because the UT is not able to send/receive data from $j$;

- the simulated position of a UT $i$ is such that the realized data rate between $i$ and the assigned AP $j$ is indeed lower than the one considered in the model solution, and this may lead to an increase of the airtime and a violation of the corresponding capacity constraint, causing unfeasibility.

To overcome this problem, we have proposed a reallocation algorithm, detailed in Algorithm 2, to modify the UT-AP association defined by the robust model solution. In the algorithm, such UT-AP associations are expressed by the variables $x_{i j}$, while $a_{j}$ denotes the airtime of $\mathrm{AP} j$, for each $j$, according to the definition provided in Section 3. At a high level, the reallocation algorithm is composed of two phases for each AP $j \in \mathcal{J}$ : deallocation (lines 2-14) and reallocation (lines 15-30). In the deallocation phase, the procedure aims at determining a set of UTs to be deallocated from $j$, and it is based on some simulation tests on the user positions. After the deallocation, the algorithm starts the reallocation phase, where a new AP is associated with each deallocated UT.

More in detail, for each AP $j \in \mathcal{J}$, the set $D U(j)$ of UTs to be deallocated from $j$ is composed of two subsets, $D U_{0}(j)$ and $D U_{1}(j)$ (see line 14). $D U_{0}(j)$ is the set of UTs $i$ having a data rate equal to 0 with respect to their simulated positions. Hereafter the data rate of user $i$ with respect to $j$ when considering the simulated position of $i$ will be denoted as $r_{i j}^{*}$ (line 3 ). $D U_{1}(j)$, instead, is a set of UTs that should be deallocated from $j$ in order to satisfy the capacity constraint of $j$. This set is defined by considering the UTs consuming higher airtime resources of $j$, as better clarified in lines 5-13 of Algorithm 2. After the definition of $D U(j)$, the algorithm starts the reallocation phase, where a new AP is associated with each UT, say $i_{c}$, in $D U(j)$. To this aim, for each considered $\mathrm{UT} i_{c}$, the algorithm calculates the set $A f U\left(i_{c}\right)$ of APs $j$ with $r_{i_{c} j}^{*}>0$ (line 18). Then, for each AP $j_{s} \in A f U\left(i_{c}\right)$, the test for evaluating if $i_{c}$ can be associated with $j_{s}$ consists in checking the capacity constraint of $j_{s}$ in case the association $\left(i_{c}, j_{s}\right)$ is established. If the capacity constraint is satisfied, then $i_{c}$ is reallocated to $j_{s}$ (lines 23-25), otherwise the algorithm considers the next $\mathrm{AP}$ in the set $A f U\left(i_{c}\right)$ (lines 26-27). The APs $j_{s}$ in $A f U\left(i_{c}\right)$ are considered according to a nonincreasing ordering of the simulated data rate $r_{i_{c} j_{s}}^{*}$ (line 19). The reallocation algorithm works in $O(|\mathcal{I}||\mathcal{J}|+|\mathcal{I}| \log |\mathcal{I}|+|\mathcal{J}| \log |\mathcal{J}|)$ time due to the two nested sorting procedures.

In our computational experience, both the powering-on heuristic and the reallocation algorithm added a contribution to the computational time which is negligible with respect to the time required to solve the robust models alone.

\section{Notes on implementing GWLANs}

Here we describe how the robust optimization approaches presented in this work can be effectively deployed in actual GWLANs, thus being of practical use. 


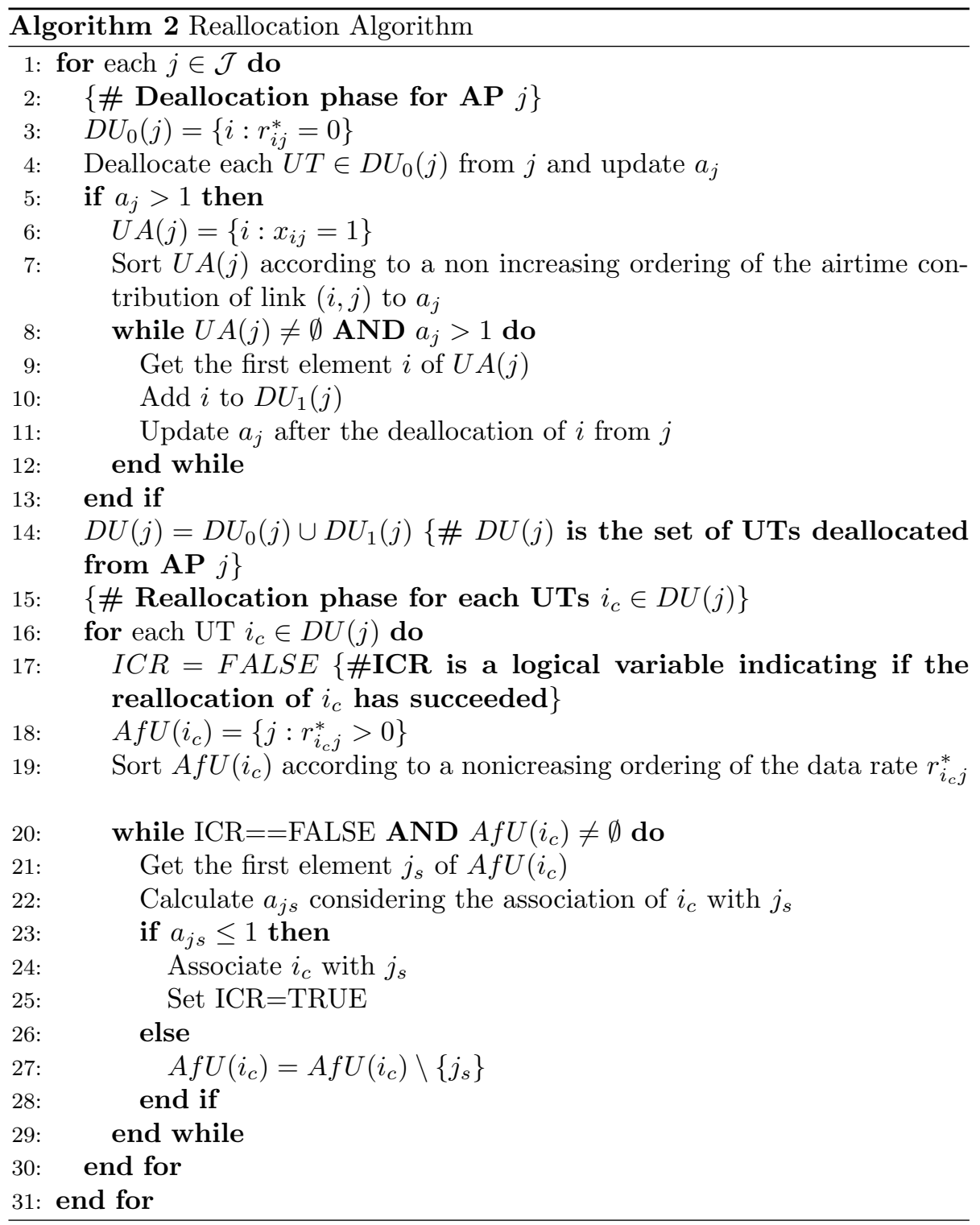


Given a Wi-Fi network, the association mechanism employed by the 802.11 standard IEEE (2016) is the strongest signal first (SSF), according to which each UT is associated with the AP from which the highest RSSI is measured. The approach is simple and completely distributed, but it does not allow to perform some optimization actions (e.g. throughput, reliability or energy efficiency maximization) which are necessary in dense Wi-Fi networks. Indeed, in dense Wi-Fi networks there are usually multiple candidate APs for each UT in a given location. In this scenario, using a simple RSSI metric may thus result in an unbalanced load of the APs and in an unfair resource allocation to the UTs. To overcome this inconvenient, recent studies have been focused on the definition of architectures for centralizing the monitoring and the control of Wi-Fi network operations, following the Software Defined Network (SDN) approach. The research activities on Software Defined WLAN (SDWLAN) have produced a set of architectures that can be used for implementing, in actual Wi-Fi networks, the control mechanisms resulting from the chosen optimization actions. The reader can refer to Dezfouli et al. (2019) for a deep and updated survey of the proposed architectures and central control mechanisms for SDWLANs. Referring to this survey, the proposed schemes can be deployed in actual networks by using, for example, the EmPower (Riggio et al. (2015)), the Odin (Suresh et al. (2012)) or the OpenSDWN (Schulz-Zander et al. (2015)) architecture. In these architectures, the controller makes the association decisions and enforces the UTs to apply them. Therefore, he can impose the UT-AP association decisions resulting from the proposed optimization approaches, aimed at minimizing the overall power consumption of the network while satisfying the robustness constraints.

In order to determine a solution, the proposed optimization approaches require information on the traffic and the location of the considered UTs, where the last information is used for estimating the available data rate on all the wireless links of the network. Depending on the selected SDWLAN architecture, some specific functions can be designed and implemented for acquiring these two input data from each UT. For example, the information on the UT location can be obtained by transmitting to the controller the measured RSSI for each AP or by means of localization mechanisms, such as the ones in (Chen et al. (2017)). But the measurement of these data may be subject to errors. This is why robust approaches to GWLANs have been proposed in this work, to cope with possible errors in the estimation of the user positions and of the radio channel quality, which are necessary for devising an optimal UT-AP association.

As described, the proposed approaches control the UT-AP association, in a robust way, with the aim of containing power consumption. Precisely, this is achieved by powering-off some of the existing APs. From an implementation perspective, each AP can be powered-on or powered-off directly by the controller by using the most suitable approach to the adopted SDWLAN architecture. For example, in the EmPOWER architecture, each AP is equipped with an Energino (Riggio et al. (2013)) add-on, which is an open toolkit for energy monitoring. This add-on provides REST-based APIs that enable the network controller to turn-on and turn-off the APs. 
As a final note, observe that a dense WLAN is commonly designed by considering the peaks of the traffic offered by the UTs. In this condition, most (probably all) the existing APs need to be powered-on in order to satisfy the service requests. However, several low traffic periods may occur (e.g. at night or considering non working days), during which most of the APs are usually underutilized, i.e. the average number of UTs per AP is low. This is the most relevant scenario for optimizing a dense WLAN, indeed. And in fact, the computational results in Section 7 show the ability of the proposed robust optimization approaches in achieving a significant power saving exactly in this context, i.e. when the load is scarce. On the other hand, as the AP utilization increases, then the gained power saving tends to become negligible, since a limited number of APs can be turned-off in order to satisfy the increased UT demand.

\section{Performance analysis}

The proposed optimization approaches to RGWLAN have been evaluated according to the following experimental plan. In the first phase of the experimentation we solved the mathematical models to RGWLAN, presented in Section 4, and the model to GWLAN, described in Section 3, using the state-of-the-art optimization solver CPLEX. The aim was to compute the optimal power consumption of the system, or a suitable approximation of it, under the uncertain and the certain data rate environment under study, respectively. Specifically, we solved the models under different settings of key CPLEX parameters, to determine a promising setting to be used in the next phase of the computational experience. Then, in the second phase we studied the performance of the proposed matheuristics by testing the impact of the powering-on heuristic and of the reallocation algorithm on the model solution. This test has been conducted in cascade. Firstly we analyzed the impact of the powering-on heuristic alone on the power consumption and on the computational time. Regarding the achieved power consumption, we compared it both with the one computed by the robust models solved without any heuristic tool, which is theoretically the minimum achievable power, and also with a straightforward upper bound. Then we considered the robust models equipped with both the powering-on heuristic and the reallocation algorithm, and we analyzed both the quality of the returned solutions, expressed in terms of power consumption and guaranteed level of robustness, and also the efficiency of the approach, expressed in terms of required computational time. Since no benchmark algorithms to RGWLAN exist in the literature, we evaluated the level of robustness of the computed solutions against the data rate uncertainty by randomly perturbing the UT positions in the reference area, and verifying the solution feasibility after the perturbation. The performance analysis has been carried out on a pool of instances which will described next. All the models have been solved by IBM ILOG CPLEX IBM 12.7.1 installed on a 64 bit Ubuntu OS, running on a $1.7 \mathrm{GHz}$ Intel Core i7 processor and equipped with $4 \mathrm{~GB}$ of memory. 


\subsection{The instances}

The network scenarios have obtained by randomly distributing UTs and APs in a square area having a side of $100 \mathrm{~m}$. This strategy is often used since it allows one to reduce the number of the scenario parameters which may influence the computational results, by permitting to analyze the algorithm behavior under a quite fair and general perspective. Notice, in fact, that since the AP and the UT locations are randomly distributed in the reference area, then rather arbitrary network scenarios and coverage areas can be addressed.

The generated scenarios are partitioned into five configurations, which differ each other in terms of the following two parameters:

- $\frac{|\mathcal{I}|}{|\mathcal{J}|}$, which represents the average number of UTs per AP; we call this parameter the UTs to AP Ratio $(U A R)$, and we consider two different values of UAR, i.e. 10 and 50, also denoted as low UAR and high UAR, respectively;

- $\frac{|\mathcal{J}|}{m^{2}}$, which is the density of the APs over the area; we consider three different settings: 1) low density configurations, where this parameter is set to $0.001,2)$ medium density configurations, by setting $\frac{|\mathcal{J}|}{m^{2}}=0.005$, and high density configurations, where $\frac{|\mathcal{J}|}{m^{2}}=0.01$.

These settings have been suggested by some experimental analyses of actual networks in different Wi-Fi deployment scenarios, such as university campus (Debele et al. (2015), Henderson et al. (2008)), corporate (Balazinska and Castro (2003)) and urban (Afanasyev et al. (2010)).

Regarding the density of the APs, the highest density is reported in Debele et al. (2015) paper for some areas of the Politecnico di Torino (PoliTo), where the authors observed a density of $0.02\left[|A P| / \mathrm{m}^{2}\right]$. The other works report a higher number of APs but distributed over larger areas, so that the density is 0.0008 $\left[|A P| / m^{2}\right]$ (Henderson et al. (2008)) or lower. According to such measurements, in our computational study the highest AP density was therefore set to 0.01 $\left[|A P| / m^{2}\right]$, i.e to a value of the same order of magnitude of the highest density in Debele et al. (2015).

To set up the UAR values, we have analyzed the actual scenarios mentioned before. The measurements carried out before 2010 highlight UAR values of a few units. However, the more recent study carried out at PoliTo highlights higher values of UAR. In particular, Figure 1 in (Debele et al. (2015)) reports the number of observed UTs in a study room with 3 APs during a weekday of the teaching period. The figure points out the presence of four phases during the day.

- Idle phase, between 21:00 to 7:00 of the day after. No user is in the room, actually the Campus is closed.

- Ramp-up transient phase, between 7:00 to 10:00. The number of UTs in the rooms grows quickly up to its typical value. However, at 9:00 the number of UTs is about $30(\mathrm{UAR}=10)$. 
- Steady-state phase, between 10:00 to 18:00. The number of UTs varies around its typical value; an average value of about 100 (UAR=33.33) with peaks of $140(\mathrm{UAR}=46.67)$ is observed.

- Emptying transient phase, between 18:00 to 21:00. The number of UTs decreases. At 19:00 they are less than $30(\mathrm{UAR}=10)$.

These results from the literature suggested us to experiment 10 and 50 as reasonable values for the parameter UAR.

Table 2 reports the relation between configuration identifiers and parameter settings. It is worth noting that the odd configurations have a low UAR, i.e. $U A R=10$ (in bold), whereas the even ones are high UAR configurations, i.e. $U A R=50$. The last column of the table reports an upper bound to the maximum power consumption of each configuration. Since the worst situation for the power consumption verifies when each AP is powered-on and works at the highest possible load, i.e. its "airtime" is equal to 1, then the upper bound can be computed according to $\sum_{j \in \mathcal{J}}\left(p_{j}^{o}+p^{w}\right.$ ) (see Section 3). In our experimentation $p_{j}^{o}$ and $p^{w}$ have been set equal to $24 W$ and $11 W$, respectively.

Table 2: Configurations: parameter settings

\begin{tabular}{|c|c|c|c|c|c|}
\hline Conf. ID & $|\mathcal{I}|$ & $|\mathcal{J}|$ & UAR & AP density & Max. Power (W) \\
\hline $\mathbf{1}$ & 100 & 10 & $\mathbf{1 0}$ & 0.001 & 350 \\
\hline 2 & 500 & 10 & 50 & 0.001 & 350 \\
\hline $\mathbf{3}$ & 500 & 50 & $\mathbf{1 0}$ & 0.005 & 1750 \\
\hline 4 & 2500 & 50 & 50 & 0.005 & 1750 \\
\hline $\mathbf{5}$ & 1000 & 100 & $\mathbf{1 0}$ & 0.01 & 3500 \\
\hline
\end{tabular}

For each configuration, fifty different instances have been generated, having the same $\frac{|\mathcal{I}| \mid}{|\mathcal{J}|}$ and $\frac{|\mathcal{J}|}{m^{2}}$ settings characterizing the configuration. In each instance, the random distribution of the APs and the UTs locations has been determined as follows. Firstly, we divided the test field into a regular grid of $|\mathcal{J}|$ squares. Then, the APs have been placed one per square, with their coordinates chosen randomly within the square. The set of the UTs has been also split into $|\mathcal{J}|$ subsets, and the elements of each subset have been randomly spread over the corresponding square. This strategy ensures enough uniformity in the placement of UTs and APs, so as to mimic a corporate scenario and to avoid heavily unbalanced instances.

Concerning the parameters related to the uncertainty in the user position, $K$, which is common to both robust models, has been set to $0.5|\mathcal{I}|$. Moreover, in the case of the Multiband Robust model we considered $|B|=4$ and $H_{b}=$ $0.25|\mathcal{I}| \forall b \in B$, while for the Basic Robust model we set $H=0.75|\mathcal{I}|$. This choice allowed us to compare the performance of the two robust models within a uniform user position uncertainty setting, since $H=H_{1}+H_{2}+H_{3}$. Furthermore, by considering the parameters $r_{i j}^{\min -b}$ in the robust capacity constraints (6), 
representing the worst value assumed by the data rate function $r_{i j}\left(b, f_{L}\right)$ for $\mathrm{UT} i$ and band $b(b \geq 1)$ with respect to the AP $j$, they have been configured according to the following procedure: for each UT $i$ and for each band $b, b \geq 1$, 3 positions of $i$ within the annulus corresponding to $b$ have been generated by using a uniform distribution; then for each $j, r_{i j}^{m i n-b}$ has been set to the minimum data rate of link $(i, j)$ determined by these generated 3 alternative positions of $i$.

Concerning the fading uncertainty, we experimented the lower end $f_{L}=0.05$. The path loss of the links has been computed by using a simplified version of the COST-231 multi-wall path loss model for indoor, NO-LOS environments $(\mathrm{Eu}-$ ropean Commission (1999)), and the maximum achievable rate set to $54 \mathrm{Mbps}$ according to the $802.11 \mathrm{~g}$ standard. Finally, the traffic demands $w_{i}$ have been generated by independent random variables, uniformly, in the range $[270,300]$ Kbps.

\subsection{Analysis of CPLEX settings}

The first phase of our computational study was devoted to solve the mathematical models to RGWLAN and to GWLAN by means of a commercial optimization solver, in order to assess at what extent they are able to return optimal solutions, or good approximations, in a time that is acceptable from an engineering perspective (see D'Andreagiovanni et al. (2017)). This has been carried out by considering different settings of the solver parameters. The aim was to determine a promising setting of such parameters, to be used in the next phase of the computational study, devoted to the matheuristic experimentation. Specifically, the following CPLEX parameters have been considered:

- the relative MIP gap tolerance (parameter CPX_PARAM_EPGAP, denoted as $E G$ in the following), which sets a relative tolerance on the gap between the best integer objective value and the objective value of the best node remaining (best bound hereafter). It instructs CPLEX to stop as soon as the ratio |best_bound-best_integer_value|/|best_integer_value| falls below the value of this parameter;

- the maximum time, denoted as $T L$ (Time Limit), given to the solver for the model solution.

$T L$ refers to the elapsed time, in seconds, since the solver starting time. That is, the solver stops its execution after $T L$ seconds, unless it computes an optimal solution before. Notice that, since CPLEX checks the elapsed time only at certain points in its code, the computational time could be a little bit higher than the set $T L$ value.

After some preliminary tests, three alternative settings have been considered. Two of them have the default $E G$ value (i.e., $\left.10^{-4}\right)$ and differ in $T L(T L=200 \mathrm{~s}$ and $T L=1800 \mathrm{~s}$, respectively). The third setting associates $T L=1800 \mathrm{~s}$ with the larger relative tolerance $E G=0.05$. Table 3 shows the summary of the 
results obtained for the 50 instances of configuration 5 , i.e. the one with the highest density, for the three models: the one disregarding the uncertainty aspects (referred to as $N O$, standing for nominal, hereafter), the Basic Robust model ( $B R$ hereafter), and the Multiband Robust model ( $M R$ in the following). Configuration 5 , in fact, proved to be the most critical for all models. Anyway similar results have been obtained with the other configurations. When the EG value is not reported, the results have been obtained by using its default value, i.e. $10^{-4}$. The table reports the following statistics: the average power consumption $(\mathrm{PC})$, the average computational time $(\mathrm{CT})$, and the average number of powered-off APs $\left(N_{O F F}\right)$. For each statistic, the average value is with respect to the number of instances for which the solver determined an optimal or a feasible solution. At this regard, the solver can terminate with three different outputs:

- optimal solution;

- feasible solution: the solver was not able to compute an optimal solution within $T L$ (or to certify the optimality), and returns the best solution found;

- no feasible solution: the solver did not find any feasible solution within $T L$.

Rows Opt., Feas., and Nofeas. in Table 3 report the number of instances for which the computation ended with an optimal solution, a feasible solution, or no solutions, respectively.

Table 3 highlights two key aspects. The first one concerns the similar performance shown by the nominal and by the Multiband Robust models. The second one concerns the performance of these two models, for $T L=1800 \mathrm{sec}-$ onds, using the two different $E G$ settings, i.e. $10^{-4}$ and 0.05 . The comparison shows that, relaxing $E G$ to 0.05 , that is allowing the solver to determine a solution with a power consumption which is not the minimal one, then there is just a negligible worsening in terms of power savings loss, with a reduction of the computational time, however, to the order of 200 seconds. Precisely, there is an increment of $\mathrm{PC}$ of just about the $0.6 \%$ with respect to the minimum possible power consumption. Furthermore, we can observe that the PC obtained by solving these two models with $T L=200$ seconds is only slightly higher than the one obtained with $T L=1800$ seconds. Our conclusion is that, for the nominal and the Multiband Robust models, after 200 seconds CPLEX has already determined a solution which is close to the optimal one, as the analysis of the indicator $O p t$. testifies.

A completely different behaviour is observed for the Basic Robust model. Indeed, in this case the average $\mathrm{PC}$ of the solutions obtained by setting $T L=$ 200 seconds is more than $23 \%$ greater than the one obtained for $T L=1800$ seconds. Furthermore, considering the relaxed setting $E G=0.05$ does not provide notable differences in the performance.

By considering the two main performance parameters, i.e. $C T$ and $P C$, we can therefore summarize the obtained results as follows: 
Table 3: Performance results for configuration 5

\begin{tabular}{|c|c|c|c|c|}
\hline & $\mathrm{PP}$ & $T L=200 \mathrm{~s}$ & $T L=1800 \mathrm{~s}$ & $T L=1800 \mathrm{~s} E G=0.05$ \\
\hline \multirow{6}{*}{ ○ } & CT (s) & 200.18 & 1800.45 & 224.71 \\
\hline & $\mathrm{PC}(\mathrm{W})$ & 527.97 & 518.434 & 521.70 \\
\hline & $N_{O F F}$ & 84.78 & 85 & 85 \\
\hline & Opt. & $\overline{0}$ & $\overline{0}$ & 50 \\
\hline & Feas. & 50 & 50 & 0 \\
\hline & No feas. & 0 & 0 & 0 \\
\hline \multirow{6}{*}{$\stackrel{n}{n}$} & CT (s) & 200.88 & 1800.65 & 1800.76 \\
\hline & $\mathrm{PC}(\mathrm{W})$ & 881.96 & 716.441 & 716.44 \\
\hline & $N_{O F F}$ & 69.82 & 76.74 & 76.74 \\
\hline & Opt. & 0 & 0 & 0 \\
\hline & Feas. & 50 & 50 & 50 \\
\hline & No feas. & 0 & 0 & 0 \\
\hline \multirow{6}{*}{$\stackrel{\vartheta}{\Sigma}$} & $\overline{\mathrm{CT}(\mathrm{s})}$ & 200.35 & 1800.87 & 282.62 \\
\hline & $\mathrm{PC}(\mathrm{W})$ & 529.46 & 518.672 & 521.97 \\
\hline & $N_{O F F}$ & 84.62 & 84.98 & 84.98 \\
\hline & Opt. & 0 & 0 & 49 \\
\hline & Feas. & 50 & 50 & 1 \\
\hline & No feas. & $\overline{0}$ & 0 & 0 \\
\hline
\end{tabular}

- $C T$ : there is a notable difference between the Multiband and the Basic Robust models. The former improves $C T$ by suitably acting on the setting of the CPLEX parameter $E G$. In fact, when $E G=0.05$, it is able to compute an optimal solution in the $98 \%$ of the cases, with an average time which is not too far from the one of the nominal model. The Basic Robust model, instead, does not show any benefit in terms of $C T$. It never finds an optimal solution within the given time limit, always reaching $T L$.

- $P C$ : for the Basic Robust model, a higher $T L$ allows one to decrease this indicator, i.e. to reduce the power consumption. Table 3 highlights in fact a PC increase of about $23 \%$ in case of $T L=200$ seconds with respect to $T L=1800$ seconds. This result is due to the significant larger number of powered-off APs obtained when $T L=1800$ seconds. In contrast, the Multiband Robust and the nominal models return $P C$ values which are similar in all the three considered settings.

Due to the above results, the performance analysis of the proposed matheuristics will be carried out by considering the CPLEX settings $T L=200$ seconds and $E G=10^{-4}$. In fact, the presented results show that this setting allows one to achieve a good trade-off between computational time and power saving. In particular, the power consumption of the robust model solutions is near to 
the minimum one, especially for the MR model, and therefore it will be used to evaluate the quality of the matheuristic solutions in terms of energy saving.

\subsection{Impact of the powering-on heuristic}

We first considered the mathematical models equipped with the powering-on heuristic alone, to analyze the trade-off between increase of the power consumption and reduction of the computational time.

As described in Section 5.1, the powering-on heuristic is based on the selection of a set of APs, $\mathcal{J}_{O N}$, which are considered as key network design elements, in the sense that they should be constantly powered-on. Specifically, $\mathcal{J}_{O N}$ is composed of the APs which are able to offer a non null data rate to at least the $80 \%$ of the UTs. Hence, when equipped with the powering-on heuristic, CPLEX searches a solution such that $y_{j}=1, \forall j \in J_{O N}$. Obviously, this strategy may cause an increase of the power consumption $(P C)$ with respect to the optimal one, since the powering-off of the APs in $\mathcal{J}_{O N}$ is forbidden. The models equipped with the powering-on heuristic thus represent mathematical based heuristics, or matheuristics, to RGWLAN, due to the forced powering-on of the APs in $\mathcal{J}_{O N}$.

Table 4 shows the effects of the powering-on heuristic on the output of the solver. The entries of the table give in fact, for each model, the number of optimal or feasible solutions found, and the number of the cases where no solution was found. The columns marked with $H$ refer to the models using the poweringon heuristic before calling the solver, i.e. the matheuristics mentioned before. It is worth highlighting that, for the columns marked with $H$, Opt. indicates optimality for the model equipped with the powering-on heuristic, i.e. for the scenarios where the APs belonging to the set $\mathcal{J}_{O N}$ are not considered in the search of the solution with minimum power because they are always poweredon. In other words, these solutions are not necessarily optimal for the exact models, where all the APs can be powered-off.

The table shows that, for the last three configurations (i.e., 3, 4 and 5), the solver is not able to determine an optimal solution for the mathematical models NO, BR and MR within the stated $T L$. For the low density configurations (i.e., 1 and 2), CPLEX returns either optimal or feasible solutions for the nominal model. For BR and MR, instead, CPLEX provides an optimal solution or it is unable to find any feasible solution within $T L$. In particular, considering the Basic Robust model, in case of configuration 2 the solver does not find any feasible solutions for none of the 50 instances. The recourse to the powering-on heuristic allows one to modify this situation. By observing the results related to the configurations with a low $U A R$, i.e. $U A R=10$ (configurations 1,3 and 5 ), we can observe that an optimal solution of model MR equipped with the powering-on heuristic is obtained for almost all the instances. In particular, in the case of the highest density configuration, i.e. configuration 5, the solver is able to find an optimal solution of the matheuristic, within the imposed time limit, for the $94 \%$ of the instances. On the other hand, the powering-on heuristic has negligible effects on the performance of the Basic Robust model 
Table 4: Comparison between mathematical models and matheuristics: type of determined solutions

\begin{tabular}{|c|c|c|c|c|c|c|c|}
\hline Conf.Id & Model & Opt. & Opt.H & Feas. & Feas.H & Nofeas. & Nofeas.H \\
\hline \multirow{3}{*}{1} & MR & 48 & 48 & 0 & 0 & 2 & 2 \\
\cline { 2 - 9 } & BR & 22 & 22 & 0 & 0 & 28 & 28 \\
\cline { 2 - 8 } & NO & 50 & 50 & 0 & 0 & 0 & 0 \\
\hline \multirow{3}{*}{2} & MR & 35 & 35 & 0 & 0 & 15 & 15 \\
\cline { 2 - 8 } & BR & 0 & 0 & 0 & 0 & 50 & 50 \\
\cline { 2 - 9 } & NO & 40 & 39 & 10 & 11 & 0 & 0 \\
\hline \multirow{3}{*}{3} & MR & 0 & 50 & 50 & 0 & 0 & 0 \\
\cline { 2 - 8 } & BR & 0 & 7 & 50 & 43 & 0 & 0 \\
\cline { 2 - 8 } & NO & 0 & 23 & 50 & 27 & 0 & 0 \\
\hline \multirow{3}{*}{4} & MR & 0 & 0 & 50 & 50 & 0 & 0 \\
\cline { 2 - 8 } & BR & 0 & 0 & 50 & 50 & 0 & 0 \\
\cline { 2 - 8 } & NO & 0 & 0 & 50 & 50 & 0 & 0 \\
\hline \multirow{3}{*}{5} & MR & 0 & 47 & 50 & 3 & 0 & 0 \\
\cline { 2 - 8 } & BR & 0 & 0 & 50 & 50 & 0 & 0 \\
\cline { 2 - 8 } & NO & 0 & 3 & 50 & 47 & 0 & 0 \\
\hline
\end{tabular}

which, requiring a high computational time, returns optimal solutions only in a few cases.

Table 5 shows the impact of the powering-on heuristic on the power consumption, i.e. $P C$, and on the computational time, i.e. $C T$. Again, the columns marked with $H$ refer to the use of the powering-on heuristic, i.e. to the matheuristics. Considering configuration 2, the solver was unable to find a feasible solution for model BR, with and without the powering-on heuristic, for all the 50 instances. Hence, no performance parameter is available, and this is indicated by symbol "-" in Table 5 .

In the table:

- "TL" in columns CT indicates that the solver reached the time limit for all the 50 instances, without returning an optimal solution;

- the columns "N.S." report the number of instances for which an optimal solution was determined; furthermore, between brackets, it is indicated the number of instances for which a feasible, but not necessarily optimal, solution was determined within the time limit;

- the values in columns "PC" and "CT" have been computed by averaging the $\mathrm{PC}$ and the $\mathrm{CT}$ values for the instances where the solver gave results, i.e. either an optimal or a feasible solution has been determined. For example, if we consider MR equipped with the powering-on heuristic for configuration 1, in column "N.S. H" we read 48(0). The meaning is that 


\begin{tabular}{|c|c|c|c|c|c|c|c|c|c|c|c|c|}
\hline 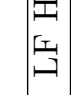 & $\Rightarrow r$ & & $p c$ & & 0 & 0 & +0 & 0 & 00 & $0 c$ & & $N$ \\
\hline 岳 & 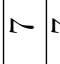 & $A$ & $b$ & & 0 & 00 & 00 & 0 & 00 & 0 & & $b 0$ \\
\hline 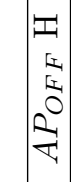 & 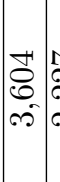 & & & & $\mid$\begin{tabular}{l}
$\infty$ \\
\hdashline \\
$-i$ \\
-1
\end{tabular} & 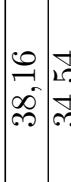 & & $\mid \begin{array}{l}0 \\
0 \\
0 \\
3 \\
-1\end{array}$ & & 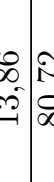 & & 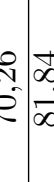 \\
\hline 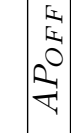 & 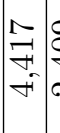 & & & & 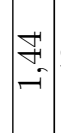 & $\mathcal{F}$ & & $\mid$\begin{tabular}{l}
4 \\
\hdashline \\
\hdashline \\
\hdashline
\end{tabular} & $\mid \begin{array}{lll}1 & b \\
0 & 0 \\
0 & 0\end{array}$ & 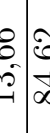 & & \begin{tabular}{l|l}
1 \\
0 \\
0 \\
0 \\
0
\end{tabular} \\
\hline $\begin{array}{l}\Psi \\
\dot{2} \\
\dot{Z} \\
\ddot{Z}\end{array}$ & $\begin{array}{c}\widehat{e} \\
\infty \\
\infty \\
+\end{array}$ & $\begin{array}{l}\hat{e} \\
\hat{v}\end{array}$ & 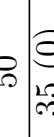 & & 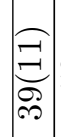 & & 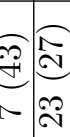 & $\begin{array}{l}0 \\
0 \\
0 \\
0\end{array}$ & 总 & $\frac{8}{8}$ & $=$ & $\begin{array}{c}b \\
b \\
b\end{array}$ \\
\hline ن & 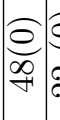 & $\begin{array}{l}e \\
\hat{v}\end{array}$ & & & 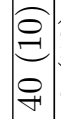 & & \begin{tabular}{l|l}
0 \\
0 \\
0 & 0 \\
0 & 0 \\
0
\end{tabular} & 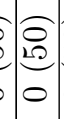 & 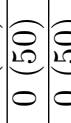 & 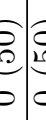 & & $\frac{2}{2}$ \\
\hline 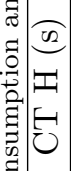 & $\vec{Z}$ & & & & 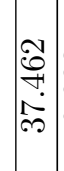 & & 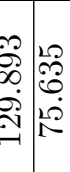 & a & & 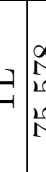 & & $=$ \\
\hline 0 & $\mid \begin{array}{l}0 \\
\stackrel{2}{0} \\
0 \\
0\end{array}$ & & & 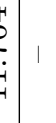 & 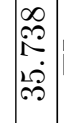 & & & 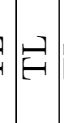 & & & & $\exists$ \\
\hline $\begin{array}{l}3 \\
Z \\
0 \\
0 \\
0\end{array}$ & 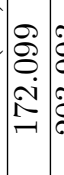 & 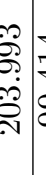 & & 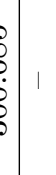 & 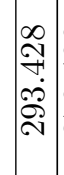 & 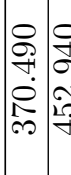 & 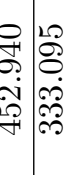 & 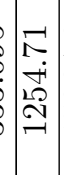 & 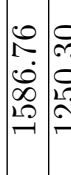 & 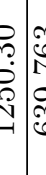 & & 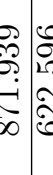 \\
\hline $\begin{array}{l}3 \\
0 \\
0\end{array}$ & 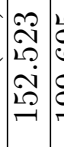 & 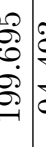 & 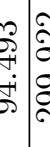 & & 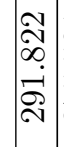 & 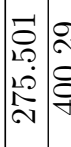 & 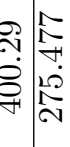 & 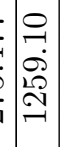 & 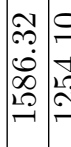 & 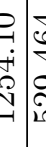 & & 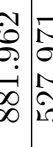 \\
\hline $\begin{array}{l}\overrightarrow{0} \\
0 \\
0 \\
0 \\
2\end{array}$ & $\sum$ & (1) & & & & & 空 & 赵 & ح) & 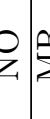 & & ح) \\
\hline 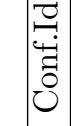 & & - & & $N$ & & & $\infty$ & & 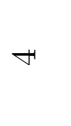 & & & مـ \\
\hline
\end{tabular}


the values reported in columns "PC H" and " $\mathrm{CT} \mathrm{H}$ " have been obtained by averaging the corresponding indicators for 48 instances (i.e. $48+0$ ). Since 50 instances have been generated for each configuration, we can deduce that, for two of them, the solver was unable to find any feasible solution within TL.

Regarding the power consumption, here a comparison is performed between the PC indicator of the matheuristics and the one of the pure mathematical models. Recall in fact that, according to the results in Section 7.2, when a feasible solution is found the selected settings of CPLEX parameters are such that a good approximation of the minimum power consumption is returned by the mathematical models in almost all the tested instances. The table highlights that MR generally provides $\mathrm{PC}$ values comparable to the ones of the nominal model, and that the use of the powering-on heuristic worsens a little its power saving performance in the configurations with $U A R=10$, i.e., 1,3 and 5 . On the other hand, when $U A R=50$, then the heuristic does not significantly change the power consumption of the returned solutions. Figure 1 helps to understand this result. In particular, the figure shows the number of poweredoff APs in the solution of MR for each of the 50 instances of configurations 3 and 4 , with and without the powering-on heuristic. Recall that these configurations are characterized by the same number of APs (i.e. 50), but have a different UAR value (10 for configuration 3 and 50 for configuration 4). We can observe that, for the instances with high UAR, the powering-on heuristic has negligible effects on the power savings. By considering the results without heuristic, when $\mathrm{UAR}=50$, given the "high" average number of UTs per AP, more than the $70 \%$ of the APs have to be powered-on in order to accomplish the UT service requirements. Therefore, in this case the number of powered-off APs presents negligible variations in all the instances, as shown by the curves referring to configuration 4. On the contrary, when $U A R=10$, the powering-on of only the $16 \%$ of the available APs allows one to satisfy the service requirements of all UTs. In this case, the performance worsening induced by the powering-on heuristic is more evident as shown by the distance between the two curves of the results related to configuration 3. At this regard, it is worth highlighting that optimizing the power consumption is a relevant issue especially when the offered traffic to the WLAN is scarce. In the considered experimentation, this condition verifies in the configurations with $\mathrm{UAR}=10$. The other considered UAR value, on the other hand, refers to a medium loaded WLAN: in this case, reasonably, the optimization can provide less benefits, and this is confirmed by the low average number of APs which are powered-off, as reported in Table 5 . Notice that, in any case, the power consumption achieved via the proposed matheuristics is very far from the upper bounds reported in Table 2 referring to the naive approach where all the APs are powered-on and work at the maximum load. This is particularly evident for the configurations with a low UAR, i.e. 1, 3 and 5 .

Concerning the computational time, the table highlights that for the high density configurations, i.e. 3,4 and 5 , the robust models alone, i.e. without the 


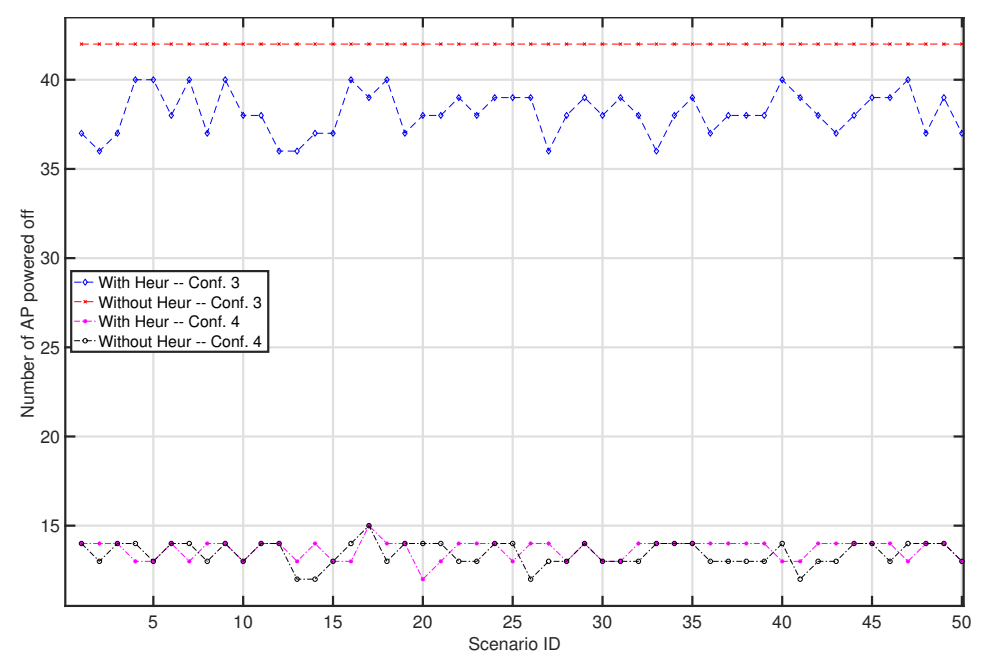

Figure 1: Number of powered-off APs for all instances of configurations 3 and 4 - MR with and without powering-on heuristic

powering-on heuristic, use all the $T L=200$ seconds for determining a model solution. The same verifies for the nominal model. The powering-on heuristic, instead, usually accelerates the model solution, at least for MR and NO and for configurations 3 and 5 . In particular, MR equipped with the powering-on heuristic is solved to optimality in most instances of configuration 3, as shown in Figure 2, The figure highlights that only 6 instances required more than 10 seconds. A numerical analysis of the results has shown that 21 instances out of 50 required less than 5 seconds for determining an optimal solution. Concerning configuration 2, characterized by $U A R=50, \mathrm{MR}$ is solved in an average time of 11.76 seconds in 35 instances, without using the heuristic. The average CT is further reduced to 6.422 seconds using the powering-on heuristic. In both cases, the solver does not find any feasible solution for the remaining 15 instances. On the other hand, in case of configuration 2 the solver is unable to find a feasible solution for BR for all 50 instances, with and without the powering-on heuristics. This result points out that, when the average number of UTs per AP is high, RGWLAN cannot be easily solved: only when the uncertainty issues are disregarded a solution is determined for all the 50 instances.

By summarizing the impact of the powering-on heuristic, it allows important reductions of the computational time especially when combined with model MR, except for the configurations 2 and 4 , which are characterized by $U A R=50$. For these two configurations, in fact, the simultaneous large number of variables and the high value of UAR make negligible the advantages in terms of CT. On the other hand, the powering-on heuristic is computationally very efficient when UAR is low, as shown by the results for configurations 1,3 and 5, with a low degradation of the power consumption with respect to the optimal, or near- 


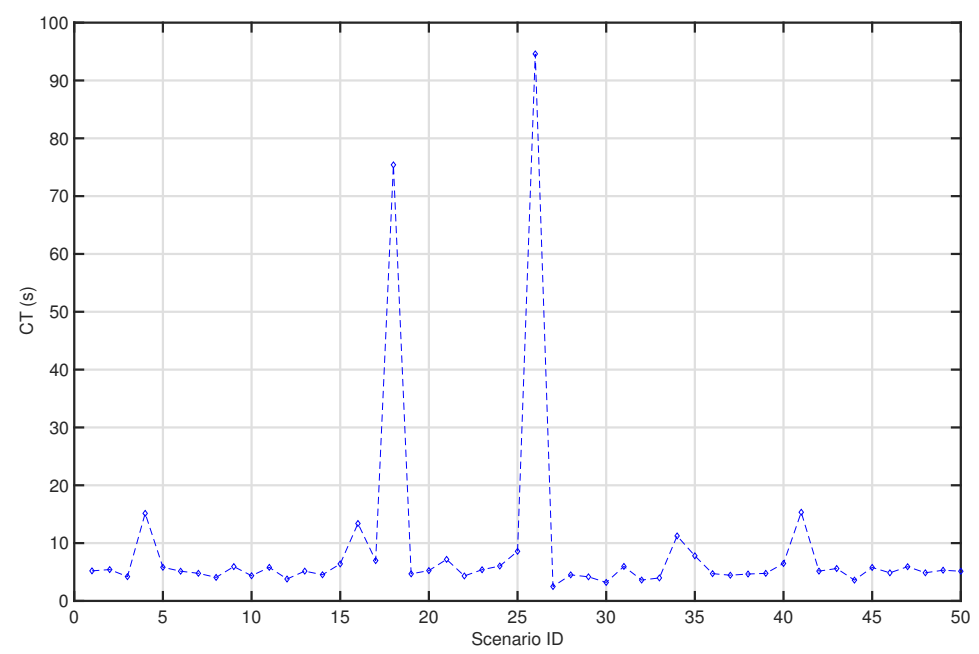

Figure 2: CT for the scenarios of configuration 3 - MR model with powering-on heuristic

optimal one, guaranteed by the robust models alone.

\subsection{Robustness analysis: impact of the reallocation algorithm}

To evaluate the robustness of the computed solutions we analyzed their feasibility with respect to a random position of the users within the annuli. Specifically, for each instance (of each configuration) we solved the robust models, with and without the powering-on heuristic. Then, we generated a companion instance, by simulating the actual position of each UT. Each companion instance has been obtained by firstly associating each UT with one band, while respecting the robustness parameters $H_{i}, i=0,1,2,3$, and then randomly generating the position of each UT within the associated annulus, using a uniform distribution. For each configuration and each approach, we thus evaluated the Level of Feasibility $(L F)$ as the number of instances for which the model solution remains feasible when applied to the companion instance, i.e. after perturbing the UT positions. As testified by the last two columns of Table 5 , the robust model solutions exhibit very low LF values for all configurations, also when the powering-on heuristic is used.

The reallocation algorithm presented in Section 5.2 has been designed to enhance the level of robustness. Applied to the instances where the solver found a solution (optimal or feasible), the idea is to give the possibility to the users to change the associated AP, to increase the number of solutions that maintain their feasibility after the user position perturbation.

Let us first analyze the impact of the reallocation algorithm when $U A R=10$. Due to the good performance shown in the previous section, only the results for the models equipped with the powering-on heuristic will be reported. The 
results for configurations 1,3 and 5 are summarized in Table 6. In the table, the acronyms have the following meaning:

- LFH: LF parameter calculated using only the powering-on heuristic;

- LFHR: LF parameter calculated using the powering-on heuristic and the reallocation algorithm: it gives the number of instances for which the solution remains feasible after applying the reallocation algorithm; the value within brackets includes the number of instances where only one UT lacks the service after the reallocation.

- ANRU: Average Number of reallocated UTs;

- ANUNS: Average Number of UTs with no service after the reallocation algorithm;

- MNUNS: Maximum Number of UTs with no service after the reallocation algorithm.

The following considerations can be drawn:

- In all cases, the number of UTs loosing their service after the reallocation is relatively low. As an example, in configuration 5, having $2500 \mathrm{UTs}$, at most 42 UTs do not have their service after the reallocation algorithm.

- By considering MR, the feasibility is guaranteed after the reallocation for almost all the instances.

- In case of MR, the average number of reallocated UTs is comparable to the one of model NO. However, the association UT-AP of the solutions obtained solving MR appears to be of better quality: by considering configuration 5, only for 13 instances the service to all UTs is not guaranteed, and only for 8 instances more than one UT losses his service.

- By considering BR, the reallocation algorithm guarantees the requested service to all the UTs in configurations 3 and 5 .

- BR shows the worst level of robustness for configuration 1 , due to the low density of the APs (only 10 APs) which does not offer enough link alternatives for guaranteeing the UT service in the simulated scenarios. This is confirmed by the results in Table 5 (column NS H), which indicate that the solver found a solution for BR only for 22 instances out of 50 .

When $U A R=50$, the results of Table 5 show that the solver did not find a solution for all the instances of configuration 2. Hence, we analyze the impact of the reallocation algorithm only for configuration 4 (see the last rows of Table 6). The results point out that the reallocation algorithm, when combined with model BR, allows one to satisfy the service requirements for all UTs in all the 50 instances. On the contrary, the reallocation algorithm shows less efficacy in case of model MR. Indeed, only for 8 instances out of 50 all the 
Table 6: Average values of some statistics before and after the application of the reallocation algorithm

\begin{tabular}{|c|c|c|c|c|c|c|}
\hline Conf.Id & Model & LFH & LFHR (only 1) & ANRU & ANUNS & MNUNS \\
\hline \multirow{3}{*}{1} & MR & $11 / 48$ & $47(48)$ & 1,29 & 0.021 & 1 \\
\cline { 2 - 7 } & BR & $7 / 22$ & $22(22)$ & 0,864 & 0 & 0 \\
\cline { 2 - 7 } & NO & $0 / 50$ & $2(10)$ & 2,48 & 3,22 & 8 \\
\hline \multirow{3}{*}{3} & MR & 0 & $45(50)$ & 7,8 & 0,1 & 1 \\
\cline { 2 - 7 } & BR & 4 & 50 & 2,5 & 0 & 0 \\
\cline { 2 - 7 } & NO & 0 & $1(2)$ & 8,98 & 8,72 & 27 \\
\hline \multirow{3}{*}{5} & MR & 0 & $37(42)$ & 19,78 & 1,64 & 24 \\
\cline { 2 - 7 } & BR & 0 & 50 & 5,42 & 0 & 0 \\
\cline { 2 - 7 } & NO & 2 & $8(16)$ & 17,5 & 11,38 & 42 \\
\hline \hline \multirow{3}{*}{4} & MR & 0 & $8(10)$ & 20,14 & 12,92 & 34 \\
\cline { 2 - 7 } & BR & 0 & 50 & 3,14 & 0 & 0 \\
\cline { 2 - 7 } & NO & 0 & $2(2)$ & 17,70 & 16,68 & 37 \\
\hline
\end{tabular}

UTs maintain the service, whereas only in two instances a single UT cannot be reallocated successfully. In case of NO, that is when robustness is not taken into account, although more than 17 UTs are on average reallocated, only in two instances the service request of all UTs is satisfied. By summarizing, when $U A R=50$ the matheuristics based on the MR model show a lower level of robustness compared to the ones based on the the Basic Robust model. This arises because the two models greatly differ in terms of switched-off APs and, as a consequence, in terms of power consumption. Referring to configuration 4, as shown in Table 6. BR works almost at full power (there are on average 0.66 APs switched-off), whereas in case of MR there are, on average, 13 APs which are switched-off (26\%). The power consumption of the solutions based on BR is thus about $26.47 \%$ higher than the average power consumption of the solutions based on MR. Such a larger power consumption, however, appears to be positive regarding the achieved level of robustness. The significant number of powered-off APs when $U A R=50$ may therefore explain the observed poor results of the MR based matheuristics in terms of level of robustness.

We conclude the section with a brief discussion of the main achievements obtained by our computational study:

- when combined with the robust model MR, the powering-on heuristic provides significant advantages in computational time when the UTs to AP ratio, i.e. UAR, is low, with a low degradation in terms of power consumption with respect to the optimal, or near optimal, power consumption guaranteed by the robust model alone; when combined with model BR, instead, the heuristic tends to power-off less APs than MR, so returning solutions with higher power consumption in a greater computational time; 
- when the UTs to AP ratio increases, the matheuristics suffer in determining solutions to RGWLAN; this is particularly true when considering model BR, which often is not able to determine a feasible solution to the problem;

- the reallocation algorithm used jointly with model BR is particularly effective on almost all the tested configurations; considering model MR, instead, it guarantees a high level of robustness when UAR is low, whereas it provides slight improvements when UAR increases.

As a final remark we emphasize that our computational results have been obtained for large network scenarios, with up to 2500 UTs and $50 \mathrm{APs}$, which correspond to the largest size of experimental results in the literature for university campus Debele et al. (2015) or corporate Balazinska and Castro (2003). However, the matheuristics proposed in this paper are applicable also to still larger campus networks composed of different buildings, such as Henderson et al. (2008), and to urban WiFi, e.g. Afanasyev et al. (2010), by partitioning the RGWLAN problem into smaller subproblems, and applying the matheuristics to each subproblem. The RGWLAN decomposition could be performed by exploiting the features of the network deployment (e.g., parts of the network having disjoint coverage areas), or defining some heuristic partitioning strategies, such as the ones preliminary investigated in D'Andreagiovanni et al. (2017) for the GWLAN problem.

\section{Conclusions}

We have proposed some heuristic algorithms to enhance mathematical models to the Robust GWLAN Problem (RGWLAN), i.e. the Basic Robust model (BR) and the Multiband Robust model (MR), thus obtaining mathematical based heuristics, or matheuristics, to RGWLAN. The aim has been to achieve a good compromise among power saving, guaranteed level of robustness and computational time required to compute a robust solution. Specifically, we have enriched the two robust models by means of: $(i)$ a preprocessing powering-on heuristic, aimed at discovering APs which it would be convenient to open, and fixing them as powered-on within the model, and (ii) a reallocation algorithm, aimed at reallocating UTs to APs once the model solution has been computed, in order to enhance the level of robustness at a negligible computational cost.

The extensive computational campaign, on 2500 instances partitioned into 5 configurations, has produced some interesting results. Specifically, when the UTs to AP ratio, denoted as $U A R$, is low, then the MR model equipped with the powering-on heuristic is computationally very efficient, with a low degradation of the power consumption with respect to the value achieved by MR alone. Also, the reallocation algorithm combined with MR guarantees a high level of robustness against the uncertainty in the user positions. When UAR increases, instead, the robust approaches are less efficient. BR shows a good level of robustness when combined with the reallocation algorithm, but it may find more 
difficulties than MR in solving the problem, by returning solutions with higher power consumption in a greater computational time. Anyway, as already outlined, optimizing the WLAN power consumption is particularly relevant when UAR is low. In this case, in fact, the offered traffic to the WLAN is scarce, and the power optimization is able to provide high benefits, as confirmed by the presented computational results.

We plan to design alternative robust heuristics to RGWLAN, and to compare them to the ones proposed in this paper on an even larger testbed, by taking into account additional sources of uncertainty such as the one related to the user demands.

\section{Acknowledgments}

This work has been partially supported by the project PRA 2017 "Modelli ed algoritmi innovativi per problemi strutturati e sparsi di grandi dimensioni" and project PRA 2018 "CONCEPT - COmmunication and Networking for vehicular CybEr-Physical sysTems" funded by the University of Pisa. Moreover, the work has been partially supported by the Italian Ministry of Education and Research (MIUR) in the framework of the CrossLab project (Departments of Excellence). The authors thank Thomas Fiorenzani for his help in the simulation work.

\section{References}

Afanasyev, M., Chen, T., Voelker, G., Snoeren, A., 2010. Usage patterns in an urban wifi network. IEEE/ACM Transactions on Networking 18, 1359-1372. doi:10.1109/TNET.2010.2040087.

Amer, M., Busson, A., Gurin Lassous, I., 2018. Association optimization based on access fairness for wi-fi networks. Computer Networks 137, 173-188. doi:10.1016/j.comnet.2018.03.004.

Archetti, C., Speranza, M., 2014. A survey on matheuristics for routing problems. EURO Journal on Computational Optimization 2, 223-246. doi:10.1007/s13675-014-0030-7.

Balazinska, M., Castro, P., 2003. Characterizing mobility and network usage in a corporate wireless local-area network, in: Proceedings of the 1st International Conference on Mobile Systems, Applications and Services, MobiSys 2003, pp. 303-316. doi:10.1145/1066116.1066127.

Bauschert, T., Büsing, C., D'Andreagiovanni, F., Koster, A., Kutschka, M., Steglich, U., 2014. Network planning under demand uncertainty with robust optimization. IEEE Communications Magazine 52, 178-185. doi 10.1109/ MCOM.2014.6736760.

Bejerano, Y., Han, S.J., Li, L., 2007. Fairness and load balancing in wireless lans using association control. IEEE/ACM Transactions on Networking 15, 560-573. doi:10.1109/TNET . 2007.893680. 
Bertsimas, D., Brown, D., Caramanis, C., 2011. Theory and applications of robust optimization. SIAM Review 53, 464-501. doi 10.1137/080734510.

Bertsimas, D., Sim, M., 2004. The price of robustness. Operations research 52, $35-53$.

Bianzino, A., Chaudet, C., Rossi, D., Rougier, J.L., 2012. A survey of green networking research. IEEE Communications Surveys and Tutorials 14, 3-20. doi:10.1109/SURV.2011.113010.00106

Burkard, R., Dell'Amico, M., Martello, S., 2009. Assignment Problems. Society for Industrial and Applied Mathematics (SIAM).

Büsing, C., D'Andreagiovanni, F., 2012. New results about multi-band uncertainty in robust optimization. Lecture Notes in Computer Science (including subseries Lecture Notes in Artificial Intelligence and Lecture Notes in Bioinformatics) 7276 LNCS, 63-74. doi $10.1007 / 978-3-642-30850-5$

Büsing, C., Grub, A., Koster, A., Laube, W., Tieves, M., 2017. Robust spectrum allocation in elastic flexgrid optical networks: Complexity and formulations. Networks 70, 342-359. doi $10.1002 /$ net.21785.

Chen, C., Chen, Y., Han, Y., Lai, H.Q., Zhang, F., Liu, K., 2017. Achieving centimeter-accuracy indoor localization on wifi platforms: A multi-antenna approach. IEEE Internet of Things Journal 4, 122-134. doi:10.1109/JIOT . 2016.2628713 .

Claßen, G., Koster, A.M., Schmeink, A., 2013. Speeding up column generation for robust wireless network planning. EURO Journal on Computational Optimization 1, 253-281.

D'Andreagiovanni, F., Garroppo, R., Scutellà, M., 2017. Power savings with data rate guarantee in dense wlans, in: 2017 International Conference on Selected Topics in Mobile and Wireless Networking, MoWNeT 2017. doi:10. 1109/MoWNet.2017.8045946.

D'Andreagiovanni, F., Garroppo, R., Scutellà, M., 2018. Green design of wireless local area networks by multiband robust optimization. Electronic Notes in Discrete Mathematics 64, 225-234. doi $10.1016 / j$.endm.2018.01.024.

Debele, F., Meo, M., Renga, D., Ricca, M., Zhang, Y., 2015. Designing resourceon-demand strategies for dense wlans. IEEE Journal on Selected Areas in Communications 33, 2494-2509. doi 10.1109/JSAC.2015.2482007

Dezfouli, B., Esmaeelzadeh, V., Sheth, J., Radi, M., 2019. A review of softwaredefined wlans: Architectures and central control mechanisms. IEEE Communications Surveys and Tutorials 21, 431-463. doi:10.1109/COMST.2018. 2868692. 
Di Puglia Pugliese, L., Guerriero, F., Poss, M., 2019. The resource constrained shortest path problem with uncertain data: a robust formulation and optimal solution approach. Computers \& Operations Research 107, 140-155.

European Commission, 1999. COST 231 - Digital Mobile Radio Towards Future Generations Systems - Final Report.

Gabrel, V., Murat, C., Thiele, A., 2014. Recent advances in robust optimization: An overview. European Journal of Operational Research 235, 471-483. doi:10.1016/j.ejor.2013.09.036.

Garroppo, R., Nencioni, G., Procissi, G., Tavanti, L., 2016a. The impact of the access point power model on the energy-efficient management of infrastructured wireless lans. Computer Networks 94, 99-111. doi $10.1016 / \mathrm{j}$.comnet. 2015.11.018.

Garroppo, R., Nencioni, G., Scutellà, M., Tavanti, L., 2016b. Robust optimisation of green wireless lans under rate uncertainty and user mobility. Electronic Notes in Discrete Mathematics 52,221-228. doi:10.1016/j.endm.2016.03. 029 .

Gendron, B., Scutellà, M., Garroppo, R., Nencioni, G., Tavanti, L., 2016. A branch-and-benders-cut method for nonlinear power design in green wireless local area networks. European Journal of Operational Research 255, 151-162. doi:10.1016/j.ejor.2016.04.058.

Henderson, T., Kotz, D., Abyzov, I., 2008. The changing usage of a mature campus-wide wireless network. Computer Networks 52, 2690-2712. doi 10 . $1016 / \mathrm{j}$. comnet.2008.05.003.

IEEE, 2016. IEEE 802.11-2016 - IEEE standard for information technologytelecommunications and information exchange between systems local and metropolitan area networks-specific requirements - part 11: Wireless LAN medium access control (MAC) and physical layer (PHY) specifications. doi:10.1109/IEEESTD.2016.7786995.

Koster, A., Poss, M., 2018. Special issue on: robust combinatorial optimization. EURO Journal on Computational Optimization 6, 207-209. doi 10.1007/ s13675-018-0102-1.

Li, G., Xu, Z., Xiong, C., Yang, C., Zhang, S., Chen, Y., Xu, S., 2011. Energyefficient wireless communications: Tutorial, survey, and open issues. IEEE Wireless Communications 18, 28-35. doi 10.1109/MWC.2011.6108331.

Li, L., Pal, M., Yang, Y., 2008. Proportional fairness in multi-rate wireless lans, in: Proceedings - IEEE INFOCOM, pp. 1678-1686. doi:10.1109/INFOCOM. 2007.154. 
Li, W., Wang, S., Cui, Y., Cheng, X., Xin, R., Al-Rodhaan, M.A., AlDhelaan, A., 2013. Ap association for proportional fairness in multirate wlans. IEEE/ACM Transactions On Networking 22, 191-202.

Riggio, R., Marina, M., Schulz-Zander, J., Kuklinski, S., Rasheed, T., 2015. Programming abstractions for software-defined wireless networks. IEEE Transactions on Network and Service Management 12, 146-162. doi:10.1109/TNSM. 2015.2417772 .

Riggio, R., Sengul, C., Suresh, L., Schulz-Zander, J., Feldmann, A., 2013. Thor: Energy programmable wifi networks, in: 2013 IEEE Conference on Computer Communications Workshops (INFOCOM WKSHPS), pp. 21-22.

Schulz-Zander, J., Mayer, C., Ciobotaru, B., Schmid, S., Feldmann, A., 2015. Opensdwn: Programmatic control over home and enterprise wifi, in: Proceedings of the 1st ACM SIGCOMM symposium on software defined networking research, p. 16.

Suresh, L., Schulz-Zander, J., Merz, R., Feldmann, A., Vazao, T., 2012. Towards programmable enterprise wlans with odin, in: Proceedings of the first workshop on Hot topics in software defined networks, pp. 115-120.

Talbi, E.G., 2016. Combining metaheuristics with mathematical programming, constraint programming and machine learning. Annals of Operations Research 240, 171-215. doi:10.1007/s10479-015-2034-y.

Tang, H., Yang, L., Dong, J., Ou, Z., Cui, Y., Wu, J., 2016. Throughput optimization via association control in wireless lans. Mobile Networks and Applications 21, 453-466. doi $10.1007 /$ s11036-015-0650-z.

Tse, D., Viswanath, P., 2005. Fundamentals of wireless communication. Cambridge university press.

Wang, X., Vasilakos, A., Chen, M., Liu, Y., Kwon, T., 2012. A survey of green mobile networks: Opportunities and challenges. Mobile Networks and Applications 17, 4-20. doi 10.1007/s11036-011-0316-4.

Wolsey, L., 1998. Integer Programming. John Wiley \& Sons.

Wu, W., Luo, J., Dong, K., Yang, M., Ling, Z., 2018. Energy-efficient user association with congestion avoidance and migration constraint in green wlans. Wireless Communications and Mobile Computing 2018. doi:10.1155/2018/ 9596141.

Zhang, Y., Jiang, C., Wang, J., Han, Z., Yuan, J., Cao, J., 2018. Green wifi implementation and management in dense autonomous environments for smart cities. IEEE Transactions on Industrial Informatics 14, 1552-1563. doi:10.1109/TII.2017.2785820 